\title{
On Hammerstein Equations with Natural Growth Conditions
}

\author{
V. Moroz and P. Zabreiko
}

\begin{abstract}
In this paper we study a nonlinear Hammerstein integral equation by means of the direct variational method. Under certain "natural" growth conditions on the non-linearity we show that the existence of a local minimum for the energy functional implies the solvability of the original equation. In these settings the energy functional may be non-smooth on its domain and, moreover, operators in data may be non-compact. Some solvability and nontrivial solvability results for the original equation are given.
\end{abstract}

Keywords: Hammerstein equations, direct variational method, non-smooth functionals

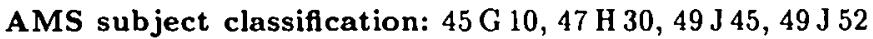

\section{Introduction}

In this paper we study the solvability of the nonlinear Hammerstein integral equation

$$
x(t)=\int_{\Omega} k(t, s) f(s, x(s)) d s
$$

where $\Omega \subset \mathbb{R}^{N}$ is a bounded domain, $k(t, s): \Omega \times \Omega \rightarrow \mathbb{R}$ is a measurable, symmetric kernel and $f(s, u): \Omega \times \mathbb{R} \rightarrow \mathbb{R}$ is a Carathéodory function. We denote by

$$
F(s, u)=\int_{0}^{u} f(s, \xi) d \xi
$$

the primitive of $f$ (note that $F(s, 0) \equiv 0$ ). In what follows $L_{p}=L_{p}(\Omega, \mathbb{R})$ stands for the Lebesgue space with the usual norm $\|\cdot\|_{L_{p}}, p^{\prime}=\frac{p}{p-1}$ for the index conjugate to $p$, and

$$
(x, y)=\int_{\Omega} x(s) y(s) d s
$$

for the standard inner product in $L_{2}$.

Both authors: State Univ., Mech.-Math. Dept., Skariny Av. 4, 220080 Minsk, Belarus moroz@mmf.bsu.unibel.by and zabreiko@mmf.bsu.unibel.by The work was supported by the Belorussian Fund of Fundamental Research. 
Equation (1) can be rewritten in the operator form

$$
x=\mathbf{K f} x
$$

where $\mathbf{f} x(s)=f(s, x(s))$ is the nonlinear superposition operator generated by the function $f$ and $\mathbf{K}$ is the linear integral operator

$$
\mathbf{K} x(t)=\int_{\Omega} k(t, s) x(s) d s
$$

generated by the kernel $k$. Hereafter we assume the following:

- $\mathbf{K}$ acts in $L_{2}$ and has the norm $\|\mathbf{K}\|$

- $\mathbf{K}$ is positive-definite on $L_{2}$, i.e. $(\mathbf{K} x, x)>0$ for all $0 \neq x \in L_{2}$.

Since the kernel $k$ is symmetric, $\mathbf{K}$ is selfadjoint in $L_{2}$ (see [10] for regular operators and [20] for the general case).

The classical direct variational approach for the treatment of the Hammerstein equation (1) goes back to the early papers by Hammerstein [7] and Golomb [6]. According to these papers the solutions of equation (1) correspond to the minimum of the energy functional $\varphi$ formed by $F$ and $\mathbf{K}$ in some Hilbert space $E$. Later, in the work by Rothe [17], Vainberg [19], Krasnosel'skii [9], Zabreiko and Povolotskii [21] the necessity of severe restrictions on the growth of $|f(s, u)|$ and "singularities" of the kernel $k(t, s)$ were clarified. More precisely, the following classical p-growth conditions

$$
\begin{aligned}
& \text { for some } p \in[2,+\infty) \text { there exist } a_{1}>0 \text { and } b_{1} \in L_{p^{\prime}} \text { such that } \\
& |f(s, u)| \leq a_{1}|u|^{p-1}+b_{1}(s) \text { and the operator } K \text { acts from } L_{p^{\prime}} \text { into } L_{p}
\end{aligned}
$$

were imposed. Under these conditions the energy functional $\varphi$ is of class $\mathcal{C}^{1}$ on $E$ and its minimum (if it exists) is a solution of the Euler-Lagrange equation $\nabla \varphi(x)=0$ which is equivalent to the original equation (1). On the other hand, a minimiser for $\varphi$ still may exist even when $\varphi$ does not possess any differentiability properties. So it is natural to ask whether it solves the Euler-Lagrange equation for $\varphi$ in some weak sense. Recently similar questions have been discussed by many authors in the context of classical calculus of variations and quasilinear elliptic boundary value problems (see $[2-5,18])$. In this paper we introduce (in some sense minimal) natural growth conditions under which the existence of a local minimum for $\varphi$ implies solvability of the Hammerstein integral equation (1). Some preliminary results of this type were obtained by the authors in [12, 14].

The presentation of the material in the paper is given in the following way. In Section 1 we define the energy space $E$ for equation (1) and study some of its properties. In Section 2 we define the energy functional $\varphi$ on $E$ and prove the existence of minimisers for $\varphi$ under the usual one-sided coercivity condition on $F$, without any additional constraints on $f$ or $\mathbf{K}$. In Section 3 we introduce the natural growth conditions under which the existence of a local minimum for $\varphi$ implies the solvability of the original Hammerstein equation (1). This allows us to obtain in Sections 4 and 5 several solvability and non-trivial solvability results for equation (1). The existence of multiple solutions for equation (1) together with some open problems in this direction are discussed in Section 6. 


\section{The energy space}

In this section we define the energy space $E$ generated by the integral operator $K$ as well as its dual space. These spaces are well known objects in the general theory of Hilbert scales generated by a linear operator (see [11]). For our further purposes we will also need some additional information about the properties of the energy space $E$ determined by specific properties of the integral operator $\mathbf{K}$.

According to our assumptions the operator $\mathbf{K}$ is bounded, selfadjoint and positive definite in $L_{2}$. Let $\mathbf{L}$ be the left inverse to $K$, that is an unbounded positive definite selfadjoint operator in $L_{2}$ with domain $D(L) \subset L_{2}$ such that $\mathbf{L K} x=x$ for all $x \in L_{2}$. We define a positive definite bilinear form on $D(L)$ by means of the formula $\langle x, y\rangle=(x, \mathrm{~L} y)$. Clearly, $\langle\cdot, \cdot\rangle$ is an inner product on $D(L)$. We define the Hilbert space $E$ as completion of $D(L)$ with respect to the norm $\|\cdot\|=\langle\cdot, \cdot\rangle^{\frac{1}{2}}$. The space $E$ is said to be the energy space for $\mathbf{K}$. We denote by $E^{\prime}$ the dual energy space obtained by the completion of $L_{2}$ with respect to the norm $\|x\|_{*}=(x, \mathbf{K} x)^{\frac{1}{2}}$.

Lemma 1. The space $E^{\prime}$ has the following properties:

(a) The conjugate to $E$ space $E^{*}$ can be identified with the space $E^{\prime}$ : for any $l \in E^{*}$ there exists $y \in E^{\prime}$ such that $l(x)=(y, x)$ for all $x \in E$; moreover, $\|l\|=\|y\|_{*}$.

(b) $E \cong \mathbf{K}^{\frac{1}{2}} L_{2} \cong \tilde{\mathbf{K}} E^{\prime}$, where $\mathbf{K}^{\frac{1}{3}}$ is the square root of $\mathbf{K}, \overline{\mathbf{K}}$ is the closure of $\mathbf{K}$ with respect to $\|\cdot\|_{*}$ and "" means an isomorphism.

(c) $\langle x, x\rangle \geq\|\mathbf{K}\|^{-1}\|x\|_{L_{2}}^{2}$ for all $x \in E$.

(d) The embeddings $E \subset L_{2} \subset E^{\prime}$ are continuous and dense.

Proof. See, for example, [11]

Let us remark that the space $E^{\prime}$ may contain some "ideal" elements and in general $E^{\prime} \not \subset S$, where we denote by $S=S(\Omega, \mathbb{R})$ the space of almost everywhere finite Lebesguemeasurable functions on $\Omega$ endowed with the topology generated by the convergence in measure. On the other hand, for the space $E$ we have the following remarkable compactness result.

Lemma 2. The embedding $E \subset S$ is continuous and compact.

Proof. The proof of the lemma follows immediately from the fact that the square root $K^{\frac{1}{2}}$ of the integral operator $\mathbf{K}$ is continuous and compact as an operator from $L_{2}$ into $S$ (see [21: Lemma 1]). Indeed, suppose that $\left(x_{n}\right) \subset E$ is a bounded sequence in $E$. By Lemma $1 /\left(\right.$ b) there exists a bounded sequence $\left(y_{n}\right) \subset L_{2}$ such that $x_{n}=K^{\frac{1}{2}} y_{n}$. Hence $\left(x_{n}\right)$ contains a subsequence converging in measure to some $x \in E$

Now suppose that we have some information about the action of the operator $\mathbf{K}$ in the scale $L_{p}$. Then we can obtain some additional information about the location of the spaces $E$ and $E^{\prime}$ with respect to the Lebesgue scale.

Lemma 3. If for some $p \in[2,+\infty)$ the operator $\mathbf{K}$ acts from $L_{p^{\prime}}$ into $L_{p}$, then the embeddings $E \subset L_{p} \subset L_{2} \subset L_{p^{\prime}} \subset E^{\prime}$ are continuous and dense.

Proof. The proof of the lemma follows from the classical Krasnosel'skii-Krein factorization theorem (see [10]) which states that the square root $\mathbf{K}^{\frac{1}{2}}$ acts from $L_{2}$ into $L_{p}$ 
and is continuous. Therefore the embedding $E \subset L_{p}$ follows immediately from Lemma $1 /(\mathrm{b})$.

Now let us consider the embedding $L_{p^{\prime}} \subset E^{\prime}$. Let $y \in L_{p^{\prime}}$. Clearly, $l(x)=(y, x)$ is a linear continuous functional on $E \subset L_{p}$ and by Lemma $1 /\left(\right.$ a) we have $y \in E^{\prime}$.

The continuity and density of the embeddings follow from the continuity of $K^{\frac{1}{2}}$ and the fact that the kernel of $K^{\frac{1}{2}}$ (in $L_{2}$ ) is trivial

\section{Existence of a minimum}

We define the energy functional on $E$ for equation (1) by the formula

$$
\varphi(x)=\frac{1}{2}\|x\|^{2}-\int_{\Omega} F(s, x(s)) d s .
$$

It is well-known (see, for example, [1]) that the requirement on $\varphi$ to be of class $\mathcal{C}^{1}$ or at least $\mathcal{C}^{0}$ on $E$ implies severe restrictions on the growth of the function $|F(s, u)|$. Moreover, without additional restriction on the growth of the primitive $F$ the functional $\varphi$ may be not well-defined on the whole space $E$. However, since $F(s, 0)=0$ by definition (and hence $\varphi(0)=0$ ) it follows that

$$
\operatorname{Dom}(\varphi)=\{x \in E:|\varphi(x)|<\infty\} \neq \emptyset .
$$

In this section we are interested in the investigation of conditions which imply the existence of a minimum for $\varphi$. According to the classical Weierstrass principle (see, for example, $[18,19])$ it suffices to verify that $\varphi$ is (sequentially) lower semicontinuous with respect to the weak topology on $E$ and coercive, that is $\varphi(x) \rightarrow+\infty$ as $\|x\| \rightarrow \infty$. Let $F$ satisfy the usual one-sided coercivity condition

$(\mathcal{A})$ There exist $a<\|\mathbf{K}\|^{-1}, b \in L_{1}$ and $y \in E^{\prime}$ such that $F(s, u)-y(s) u \leq \frac{a}{2} u^{2}+b(s)$ where $y \in E^{\prime}$ means that $l(x)=(y, x)$ is a linear continuous functional on $E$. We assert that under condition $(\mathcal{A})$ the functional $\varphi$ attains its infimum.

Lemma 4. Let the coercivity condition $(\mathcal{A})$ be satisfied. Then $\varphi$ is bounded from below and coercive.

Proof. Condition $(\mathcal{A})$ and Lemma $1 /(\mathrm{c})$ imply

$$
\begin{aligned}
\varphi(x) & \geq \frac{1}{2}\|x\|^{2}-\int_{\Omega}\left\{\frac{a}{2} x^{2}(s)+b(s)\right\} d s-\int_{\Omega} y(s) x(s) d s \\
& =\frac{1}{2}\left(\|x\|^{2}-a\|x\|_{L_{2}}^{2}\right)-\|b\|_{L_{1}}-(y, x) \\
& \geq \frac{1}{2}(1-a\|\mathrm{~K}\|)\|x\|^{2}-\|b\|_{L_{1}}-\|y\|_{*}\|x\| \\
& \rightarrow+\infty
\end{aligned}
$$

as $\|x\| \rightarrow+\infty$ 
Lemma 5. Let the coercivity condition $(\mathcal{A})$ be satisfied. Then $\varphi$ is (sequentially) lower semi-continuous in the weak topology on $E$.

Proof. The energy functional $\varphi$ can be rewritten in the form

$$
\begin{aligned}
\varphi(x)= & \frac{1}{2}\|x\|^{2}-\int_{\Omega} F(s, x(s)) d s \\
= & \frac{1}{2}\|x\|^{2}-\int_{\Omega}\left\{\frac{a}{2} x^{2}(s)+b(s)\right\} d s+\int_{\Omega} y(s) x(s) d s \\
& +\int_{\Omega}\left\{\frac{a}{2} x^{2}(s)+b(s)-\{F(s, x(s))-y(s) x(s)\}\right\} d s \\
= & \left\{\frac{1}{2}\|x\|^{2}-\frac{a}{2}\|x\|_{L_{2}}^{2}\right\}-\|b\|_{L_{1}}-(y, x) \\
& +\int_{\Omega}\left\{\frac{a}{2} x^{2}(s)+b(s)-\{F(s, x(s))-y(s) x(s)\}\right\} d s .
\end{aligned}
$$

Let us consider herein each term separately. We have by condition $(\mathcal{A})$ and Lemma $1 /(c)$

$$
\frac{1}{2}\|x\|^{2}-\frac{a}{2}\|x\|_{L_{2}}^{2} \geq \frac{1}{2}(1-a\|\mathbf{K}\|)\|x\|^{2} \geq 0 \quad \text { for all } x \in E .
$$

Hence the quadratic term is (sequentially) weakly lower semicontinuous as a positive definite form on $E$ (see [19]). The linear term $(y, x)$ is also weakly continuous on $E$.

Now let us consider the last term

$$
\psi(x)=\int_{\Omega}\left\{\frac{a}{2} x^{2}(s)+b(s)-\{F(s, x(s))-y(s) x(s)\}\right\} d s .
$$

Let $\left(x_{n}\right) \subset \operatorname{Dom}(\varphi)$ be weakly converging to $x_{0}$. Then the sequence

$$
u_{n}(s)=\frac{a}{2} x_{n}^{2}(s)+b(s)-\left\{F\left(s, x_{n}(s)\right)-y(s) x_{n}(s)\right\}
$$

converges to

$$
u_{0}(s)=\frac{a}{2} x_{0}^{2}(s)+b(s)-\left\{F\left(s, x_{0}(s)\right)-y(s) x_{0}(s)\right\}
$$

in measure. From condition $(\mathcal{A})$ it follows that the sequence $\left(u_{n}\right)$ is non-negative. Now applying the Fatou lemma we obtain

$$
\int_{\Omega} u_{0}(s) d s \leq \liminf _{n \rightarrow \infty} \int_{\Omega} u_{n}(s) d s
$$

which means that $\psi$ is (sequentially) weakly lower semicontinuous

Theorem 1. Let the coercivity condition $(\mathcal{A})$ be satisfied. Then $\varphi$ is bounded from below and attains its infimum on each non-empty weakly closed set $\mathcal{M} \subseteq E$.

Proof. The proof of the theorem follows from Lemmas 4 and 5 
Remark 1. There are many well-known results of this sort (see, for example, $[9$, 21]). In contrast to the previous results we do not need any compactness-type assumptions on the operator $\mathbf{K}$ or primitive $F$ (see [21] for the discussion of these conditions in connection with standard p-growth conditions). The "compactness" of the variational problem follows from the measure-compactness of integral operators in $L_{2}$ (Lemma 3 ).

Remark 2. The restriction $a<\|K\|^{-1}$ in the coercivity condition $(\mathcal{A})$ can be slightly relaxed using the method developed by A. M. Krasnosel'skii which is based on some delicate integral inequalities (see [8] for a discussion).

\section{Natural growth conditions}

In this section we are interested in minimal conditions on the operator $\mathbf{K}$ and nonlinearity $f$ which ensure that each point of local minimum of $\varphi$ corresponds to a solution of the Hammerstein equation (1). We say that the natural $(p, q) \cdot$ growth conditions for equation (1) are satisfied if for some $p \geq 2$ and $q \geq p$

$\left(\mathcal{A}_{1}\right) \mathrm{K}$ acts from $L_{p^{\prime}}$ into $L_{p}$

$\left(\mathcal{A}_{2}\right) \mathrm{K}$ acts from $L_{\infty}$ into $L_{q}$

$\left(\mathcal{A}_{3}\right)$ There exists $a_{1}>0, b_{1} \in L_{q^{\prime}}$ such that $|f(s, u)| \leq a_{1}|u|^{\frac{p}{q^{\prime}}}+b_{1}(s)$.

Clearly, in the case $q=p$ the conditions $\left(\mathcal{A}_{1}\right)-\left(\mathcal{A}_{3}\right)$ coincide with the classical $p$-growth conditions guaranteeing that the energy functional $\varphi$ is of class $\mathcal{C}^{1}$ on $E$. However, if $q>p$, then in general the functional $\varphi$ need not be differentiable on $E$. Moreover, in this case in general $\operatorname{Dom}(\varphi) \subset E$. Nevertheless, if we confine ourselves to a restricted class of "test functions" from $E$, it is possible to derive necessary conditions of the extremum in the form of the Euler-Lagrange equation. This class must be so small to agree with the growth of $|f(s, u)|$. On the other hand, it must be sufficiently large to ensure the "necessary conditions" for the solvability of equation (1). We will show that under the $(p, q)$-growth conditions $\left(\mathcal{A}_{1}\right)-\left(\mathcal{A}_{3}\right)$ both requirements are satisfied for $\varphi$ by the functions from the test space $T=\mathbf{K} L_{\infty}$. By condition $\left(\mathcal{A}_{2}\right)$ it follows that $T \subset L_{q}$. By Lemma $1 /(\mathrm{b})$ and (d), $L_{\infty} \subset L_{2} \subset E^{\prime}$ and hence $T=\mathrm{K} L_{\infty} \subset E$.

Lemma 6. Let the $(p, q)$-growth conditions $\left(\mathcal{A}_{1}\right)-\left(\mathcal{A}_{3}\right)$ be satisfied and $p \geq 2$, $q \geq p$. Then for any $x \in \operatorname{Dom}(\varphi)$ and each $h \in T$ there exists the variation

$$
\delta \varphi(x, h)=\left.\frac{d}{d \tau} \varphi(x+\tau h)\right|_{\tau=0}=\langle x, h\rangle-\int_{\Omega} f(s, x(s)) h(s) d s .
$$

Proof. Set

$$
\psi(x)=\int_{\Omega} F(s, x(s)) d s .
$$

Clearly, $\operatorname{Dom}(\psi)=\operatorname{Dom}(\varphi)$. By the mean value theorem for each $x \in \operatorname{Dom}(\varphi)$ and $h \in T$ there exists a function $\theta$ such that $0 \leq \theta(s) \leq 1$ and

$$
\frac{\psi(x+\tau h)-\psi(x)}{\tau}=\int_{\Omega} f(s, x(s)+\tau \theta(s) h(s)) h(s) d s .
$$


It is known [21] that the function $\theta$ may be chosen to be measurable so $\theta \in L_{\infty}$ and the right-hand side of (3) has sense.

We shall verify that the integral in the right-hand side of (3) does exist. From condition $\left(\mathcal{A}_{1}\right)$ by Lemma 3 it follows that $E \subset L_{p}$ and hence we have for $\tau \in \mathbb{R}$ that $x+\tau \theta h \in L_{p}$. Further, from condition $\left(\mathcal{A}_{3}\right)$ it follows that the superposition operator f maps $L_{p}$ into $L_{q^{\prime}}$ (see, for example, [1]) and hence $f(\cdot, x(\cdot)+\tau \theta(\cdot) h(\cdot)) \in L_{q^{\prime}}$. Since $h \in T$ it follows that $f(\cdot, x(\cdot)+\tau \theta(\cdot) h(\cdot)) h(\cdot) \in L_{1}$ that is the integral in the right-hand side of (3) does exist.

Let $|\tau| \leq 1$ and $\tau \rightarrow 0$. Clearly, $x(s)+\tau \theta(s) h(s) \rightarrow x(s)$ in measure, and there exists $u \in L_{p}$ such that $|x(s)+\tau \theta(s) h(s)| \leq u(s)$ for all $\tau \in[-1,1]$. So the Lebesgue dominated convergence theorem can be applied to (3) and we have

$$
\begin{aligned}
\delta \psi(x, h) & =\left.\frac{d}{d \tau} \varphi(x+\tau h)\right|_{\tau=0} \\
& =\lim _{\tau \rightarrow 0} \frac{\psi(x+\tau h)-\psi(x)}{\tau} \\
& =\lim _{\tau \rightarrow 0} \int_{\Omega} f(s, x(s)+\tau \theta(s) h(s)) h(s) d s \\
& =\int_{\Omega} f(s, x(s)) h(s) d s .
\end{aligned}
$$

This implies (2) since the term $\frac{1}{2}\|x\|^{2}$ is smooth on $E$

Now we proceed to the main result of the section.

Theorem 2. Let the $(p, q)$-growth conditions $\left(\mathcal{A}_{1}\right)-\left(\mathcal{A}_{3}\right)$ be satisfied and $p \geq 2$, $q \geq p$. Then each local minimum $x^{*} \in \operatorname{Dom}(\varphi)$ of $\varphi$ is a solution of the Hammerstein equation (1).

Proof. Let $x^{*} \in \operatorname{Dom}(\varphi) \subset E \subset L_{p}$ be a minimum of $\varphi$. By Lemma 6 the variation $\delta \varphi\left(x^{*}, h\right)$ with respect to $h \in T$ exists. Moreover, since $x^{*}$ is a local minimum of $\varphi$ on $E$ it follows that $\delta \varphi\left(x^{*}, h\right)=0$ for all $h \in T$. By the definition of the inner product $\langle\cdot, \cdot\rangle$ we have

$$
\delta \varphi\left(x^{*}, h\right)=\left\langle x^{*}, h\right\rangle-\int_{\Omega} f\left(s, x^{*}(s)\right) h(s) d s=\left(\mathbf{L} x^{*}, h\right)-\left(\mathbf{f} x^{*}, h\right)=0
$$

for all $h \in T$. Since $T=\mathbf{K} L_{\infty}$ and the kernel of $\mathbf{K}$ on $L_{\infty}$ is trivial the operator $\mathbf{K}$ is one-to-one between $L_{\infty}$ and $T$. Hence we can write

$$
\delta \varphi\left(x^{*}, h\right)=\left(\mathbf{L} x^{*}, \mathbf{K g}\right)-\left(\mathbf{f} x^{*}, \mathbf{K} g\right)=0 \quad \text { for all } g \in L_{\infty} .
$$

Bearing in mind that $\mathbf{f} x^{*} \in L_{q^{\prime}}$ by condition $\left(\mathcal{A}_{3}\right), \mathbf{K f} x^{*} \in L_{1}$ by condition $\left(\mathcal{A}_{2}\right)$ and the kernel $k(t, s)$ is symmetric, one can easily see that $x^{*}-\mathbf{K f} x^{*} \in L_{1}$. Hence by the definition of $\mathbf{L}$ we obtain

$$
\delta \varphi\left(x^{*}, h\right) \doteq\left(\mathbf{K L} x^{*}, g\right)-\left(\mathbf{K} \mathbf{f} x^{*}, g\right)=\left(x^{*}-\mathbf{K f} x^{*}, g\right)=0
$$

for all $g \in L_{\infty}$ or, in other words, $x^{*} \in L_{p}$ is a solution of the original Hammerstein equation (1) 
Remark 3. Clearly, our choice of $T$ as "test". space for $\varphi$ is not the only possible. For example, we can choose as "test" space some "weighted" space $L_{\infty, u_{0}}$ with weight $u_{0}$ compatible with the singularities of the kernel $k(t, s)$ or the subspace of $E$ generated by the eigenfunctions of $\mathbf{K}$ etc. (see [14] for an abstract approach and details).

\section{Existence of the solution}

Basic existence results for the Hammerstein equation (1) follow immediately from Theorems 1 and 2.

Theorem 3. Let the coercivity condition $(\mathcal{A})$ and the $(p, q)$-growth conditions $\left(\mathcal{A}_{1}\right)$ $-\left(\mathcal{A}_{3}\right)$ be satisfied and $p \geq 2, q \geq p$. Then the Hammerstein equation (1) has at least one solution in $L_{p}$.

Remark 4. The result seems to be new even in the case $q=p$ (that is the case of the classical p-growth condition). In contrast to previous results of this sort we do not need any compactness-type restriction on $\mathbf{K}$ or $f$ (cf. Remark 1).

Remark 5. Let $\mathcal{L}(\mathbf{K})$ be the set of all pairs $(\alpha, \beta) \in[0,1] \times[0,1]$ such that the operator $K$ acts from $L_{\frac{1}{\alpha}}$ into $L_{\frac{1}{3}}$. Following [10] we call $\mathcal{L}(\mathbf{K})$ the $\mathcal{L}$-characteristic of $K$. Since the kernel $k(t, s)$ is symmetric the set $\mathcal{L}(\mathbf{K})$ is always symmetric with respect to the line $\alpha+\beta=1$. Since $L_{\frac{1}{a_{1}}} \subseteq L_{\frac{1}{a_{2}}}$ for $\alpha_{1} \leq \alpha_{2}$, the set $\mathcal{L}(\mathbf{K})$ contains along with any points $(\alpha, \beta)$ the rectangle co $\{(\alpha, \beta),(\alpha, 1),(0, \beta),(0,1)\}$. Moreover, the Riesz-Thorin interpolation theorem [10] states that the $\mathcal{L}$-characteristic $\mathcal{L}(\mathbf{K})$ is always a convex set. Figures 1 and 2 present the $\mathcal{L}$-characteristic of $\mathbf{K}$ under the classical $p$-growth condition and the natural $(p, q)$-growth conditions, respectively.

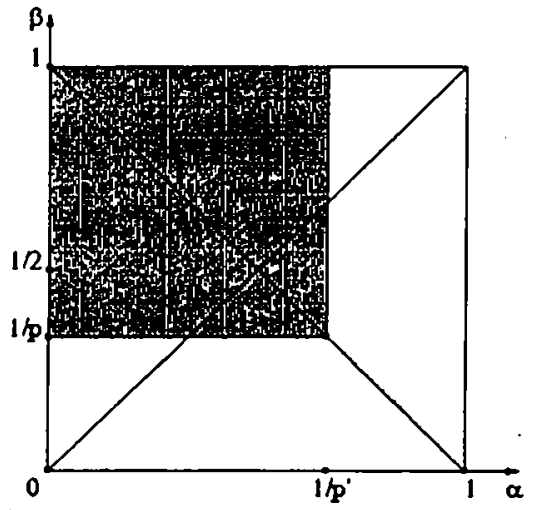

Fig.!

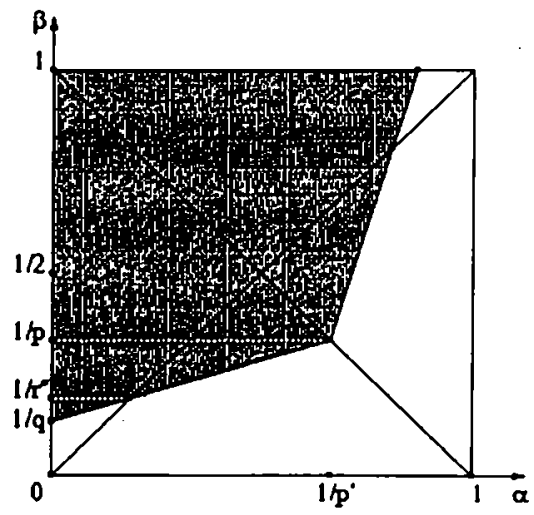

Fig. 2

Remark 6. A typical example of a function $f$ satisfying the natural $(p, q)$-growth conditions and not satisfying the classical p-growth conditions is given by

$$
f(s, u)=|u|^{\frac{p}{q^{\prime}}}+y(s)
$$


with $q>p$ and $y \in L_{\mathbf{p}^{\prime}}$. Non-linearities of a different sort are given by functions $f$ with the bounded primitive $F(s, u)=\sin \left(u v^{p}\right)$. In this case the energy functional $\varphi$ is continuous on $E$ but if $q>p$, then it is nowhere differentiable on $E$ in the classical sense. This is also the case for the primitive $F(s, u)=\sin (b(s) u)$ with $b \in L_{q^{\prime}}$ and $b \notin L_{p^{\prime}}$ but in this case the growth on $s$ is essential. However, we note that in the case of the non-homogeneous equation

$$
f(s, u)=g(s, u)-y(s)
$$

with $g(s, 0) \equiv 0$ we can not relax the restriction on the $s$-singularity of $y$. In view of condition $(\mathcal{A}), y$ should belong to $L_{p^{\prime}}$ since $L_{p^{\prime}} \subset E^{\prime}$.

As an example of an application of Theorem 3 let us consider the Dirichlet boundary value problem

$$
\left.\begin{array}{rlrl}
-\Delta u & =g(u)+y(s) & & \text { in } \Omega \\
u & =0 & & \text { on } \partial \Omega
\end{array}\right\}
$$

where $\Omega \subset \mathbb{R}^{N}$ is an open bounded domain with a smooth boundary and $g$ is a continuous function satisfying the condition $g(0)=0$. We denote by $\lambda_{1}$ the first eigenvalue of the Laplacian $-\Delta$ on $\Omega$. It is known that in the case when $y \in L_{\frac{N}{2}}$ the solutions of problem (4) belong to $L_{\infty}$. Therefore, under the one-sided condition $u g(u) \leq a u^{2}+b$ for some $a<\lambda_{1}$ and $b \in \mathbb{R}$ the special truncation procedure (based on a priory estimates for problem (4) and the weak maximum principle for $-\Delta$ ) can be applied. As a result, when using variational methods we do not need any condition on the growth of $g$ for the treatment of (4). Moreover, the energy functional corresponding to problem (4) is of class $\mathcal{C}^{1}$ (see, for example, [18]).

Let us consider the case of a singular right-hand term $y \in L_{\frac{2 N}{N+2}}$. It is known (see [10]) that problem (4) is equivalent to the Hammerstein equation (1) with the kernel $k(t, s)$ generated by the Green function for the Laplacian $-\Delta$ on $\Omega$. The classical growth conditions lead to the $\frac{N+2}{N-2}$-growth of $g$. Using Theorem 3 with $p=\frac{2 N}{N-2}$ and $q=\infty$ we obtain the following result.

Theorem 4. Let there exist $a<\lambda_{1}$ and $b \in \mathbb{R}$ such that $\int_{0}^{t} g(\xi) d \xi \leq \frac{a}{2} u^{2}+b$ and let there exist $a_{1}, b_{1} \in \mathbb{R}$ such that $|g(u)| \leq a_{1}|u|^{\frac{2 N}{N-2}}+b_{1}$. Then for each $y \in L_{\frac{2 N}{N+2}}$ problem (4) has at least one solution in the Sobolev space $\mathrm{H}_{0}^{1}(\Omega)$.

Proof. The proof ollows from Theorem 3 and the Sobolev embedding theorems

Remark 7. A similar problem for (4) was considered in [5]. Using the method of sub- and super-solutions the authors proved the existence of a solution of problem (4) in the case $y \in L_{\infty}$ under a one-sided coercivity condition $(\mathcal{A})$ and without any additional assumptions on the growth of $g$. See also $[3,4]$ or further results in this direction based on special technique of partial differential equations. 


\section{Existence of a non-trivial solution}

Henceforth we assume that $f(s, 0) \equiv 0$, so we are interested in the existence of a nontrivial solution of equation (1). More precisely, in the rest of the paper we assume that the function $f$ is of the special form

$$
f(s, u)=\alpha u+g(s, u) \quad \text { for some } \alpha \in \mathbb{R}
$$

with $g$ satisfying the condition

$$
\begin{aligned}
& \left(\mathcal{A}_{3}\right)^{\prime} \text { For each } \varepsilon>0 \text { there exists } a_{e}>0 \text { and } b_{\varepsilon} \in L_{\frac{p+q^{\prime}}{p-q^{\prime}}} \text { with }\left\|b_{\varepsilon}\right\|_{L_{\frac{p+g^{\prime}}{p-q^{\prime}}}}<\varepsilon \text { such that } \\
& \qquad|g(s, u)| \leq a_{e}|u|^{\frac{\rho}{q^{\prime}}}+b_{e}(s)|u| .
\end{aligned}
$$

This condition is stronger than condition $\left(\mathcal{A}_{3}\right)$. We rewrite the energy functional $\varphi$ in the form

$$
\varphi(x)=\frac{1}{2} \mathcal{E}_{\alpha}(x)-\psi(x)
$$

where

$$
\mathcal{E}_{\alpha}(x)=\|x\|^{2}-\alpha\|x\|_{L_{2}}^{2}, \quad \psi(x)=\int_{\Omega} G(s, x(s)) d s, \quad G(s, u)=\int_{0}^{u} g(s, \xi) d \xi .
$$

Clearly, $\mathcal{E}_{\alpha}$ is a continuous form on $E$. We will show that $\mathcal{E}_{\alpha}$ is the second variation of the energy functional $\varphi$ at zero with respect to some reasonable class of "test functions" from $E$. In contrast to the test space $T$ we do not need this class to be sufficiently large. As we will see in the following for this class it suffices to contain only the first eigenfunction of $\mathbf{K}$.

Lemma 7. Let the $(p, q)$-growth conditions $\left(\mathcal{A}_{1}\right),\left(\mathcal{A}_{2}\right)$ and $\left(\mathcal{A}_{3}\right)^{\prime}$ be satisfied and $p>2, q \geq p$. Then for each $h \in E \cap L_{\frac{p}{q}+1}$ there exists the second variation of $\varphi$ at zero

$$
\delta^{2} \varphi(0, h)=\left.\frac{d^{2}}{d \tau^{2}} \varphi(\tau h)\right|_{r=0}=\mathcal{E}_{\alpha}(h) .
$$

Proof. From condition $\left(\mathcal{A}_{3}\right)^{\prime}$ it follows that for each $\varepsilon>0$ there exists $a_{\varepsilon}>0$ and $b_{e} \in L_{\frac{p+q^{\prime}}{p-q^{\prime}}}$ with $\left\|b_{\varepsilon}\right\|_{L_{\frac{p+q^{\prime}}{p-q^{\prime}}}}<\varepsilon$ such that $|G(s, u)| \leq a_{\varepsilon}|u|^{\frac{p}{q^{\prime}+1}}+b_{\varepsilon}(s)|u|^{2}$. Hence we obtain for $h \in E \cap L_{\frac{q}{q}+1}^{p}$ (using the Hölder inequality)

$$
|\psi(\tau h)| \leq a_{\varepsilon} \tau^{\frac{p}{q^{\prime}+1}} \int_{\Omega}|h(s)|^{\frac{p}{q^{\prime}+1}} d s+\tau^{2} \int_{\Omega} b_{e}(s)|h(s)|^{2} d s \leq c_{1} a_{\varepsilon} \tau^{\frac{p}{q^{T}+1}}+c_{2} \varepsilon^{\frac{p-q^{\prime}}{p+q^{\prime}}} \tau^{2}
$$

with $c_{1}, c_{2}>0$ depending only on $h$. Since $\frac{p}{q^{\prime}}+1>2$ and $\varepsilon$ is arbitrarily small we have $\lim _{\tau \rightarrow 0} \frac{\psi(\tau h)}{\tau^{2}}=0$ which proves the lemma

Observe that in the case $p>.2$ the operator $\mathrm{K}$ acts from $L_{p^{\prime}}$ into $L_{p} \subset L_{2}$ and hence $\mathbf{K}$ is compact in $L_{2}[10]$. We denote by

$$
\lambda_{1} \geq \lambda_{2} \geq \ldots \lambda_{k} \geq \ldots>0
$$

the eigenvalues of $\mathrm{K}$ in $L_{2}$ and by $\left(e_{k}\right) \subset L_{2}$ the corresponding eigenfunctions. We will show that our assumptions imply some additional regularity of these eigenfunctions. 
Lemma 8. Let the $(p, q)$-growth conditions $\left(\mathcal{A}_{1}\right)$ and $\left(\mathcal{A}_{2}\right)$ be satisfied and $p>2$, $q \geq p$. Then $\left(e_{k}\right) \subset E \cap L_{r}$ for each $r<r^{*}=\frac{q(p-2)+p}{p-1}$.

Proof. By conditions $\left(\mathcal{A}_{1}\right)$ and $\left(\mathcal{A}_{2}\right)$, the operator $\mathrm{K}$ acts from $L_{p^{\prime}}$ into $L_{p}$ and from $L_{\infty}$ into $L_{q^{\prime}}$. The Riesz-Thorin interpolation theorem [10] states that for $\tau \in\left[p^{\prime}, \infty\right]$ the operator $\mathrm{K}$ acts from $L_{r}$ into $L_{r}$ with

$$
\frac{1}{r}=\frac{p-q}{q(p-1)} \frac{1}{\tau}+\frac{1}{q} \text {. }
$$

Let $r^{*}>2$ be the solution of the equation

$$
\frac{1}{r}=\frac{q-p}{q(p-1)} \frac{1}{r}+\frac{1}{q}, \quad \text { that is } r^{*}=\frac{q(p-2)+p}{p-1} .
$$

Hence for each $r<r^{*}$ there exists $n \in \mathbb{N}$ such that $\mathbf{K}^{n}$ acts from $L_{2}$ into $L_{r}$ (see Figure 2 for the "visualization" of the number $r^{*}$ ). Let $e_{k} \in L_{2}$ be an eigenfunction of $K$. Iterating $e_{k}$ by $\mathbf{K}$ we obtain $\left(\lambda_{k}\right)^{n} e_{k}=\mathbf{K}^{n} e_{k}$ and hence $e_{k} \in E \cap L_{r}$ for each $r<r^{*}$

Theorem 5. Let the coercivity condition $(\mathcal{A})$ and the $(p, q)$-growth conditions $\left(\mathcal{A}_{1}\right)$, $\left(\mathcal{A}_{2}\right)$ and $\left(\mathcal{A}_{3}\right)^{\prime}$ be satisfied and $p>2, q>\max \left\{p, \frac{p-1}{p-2}\right\}$. Then for each $\alpha>\lambda_{1}^{-1}$ the Hammerstein equation (1) has at least one non-trivial solution in $L_{p}$.

Proof. The existence of a minimum for $\varphi$ follows from condition $(\mathcal{A})$ by Theorem 1. Since $q>\max \left\{p, \frac{p-1}{p-2}\right\}$ we have

$$
\frac{p}{q^{\prime}}+1<r^{*}=\frac{q(p-2)+p}{p-1} .
$$

Hence by Lemma 8 the eigenfunctions of $\mathbf{K}$ belong to $L_{\frac{p}{q}+1}$ and by Lemma 7 the second variation of $\varphi$ with respect to the eigenfunctions $\left(e_{k}\right)$ does exist. Consider $e_{1}$ as a test function. We obtain

$$
\delta^{2} \varphi\left(0, e_{1}\right)=\mathcal{E}_{\alpha}\left(e_{1}\right)=\left(\lambda_{1}^{-1}-\alpha\right)\left\|e_{1}\right\|_{L_{2}}<0 .
$$

This means that zero is not a local minimum for $\varphi$ and hence $\varphi$ attains its minimum at some point $x$ different from zero. Therefore Theorem 2 implies that $x \neq 0$ is a solution of equation (1)

Remark 8. Clearly, in the statement of the theorem we have $\max \left\{p, \frac{p-1}{p-2}\right\}=p$ for $p \geq \frac{3+\sqrt{5}}{2}$, that is the additional restriction on $q$ is required only in the case of "small" p.

Remark 9. For the classical growth conditions an analogous result was established in [22] for more general non-linearities $f$ of the form $f(s, u)=a(s) u+g(s, u)$ with $a \in L_{\frac{p}{p-2}}$. In this paper we consider only the simplest case $a(s) \equiv \alpha$. The statement of Theorem 5 can be easily generalized up to the case $a \in L_{\infty}$. However, in the case of larger singularities of $a(s)$ some additional delicate control on the growth of the primitive $G(s, u)$ is required (see [13] for details). 


\section{Multiple solutions}

In this section we prove a simple multiplicity result for the Hammerstein equation (1).

Theorem 6. Let the coercivity condition $(\mathcal{A})$ and the $(p, q)$-growth conditions $\left(\mathcal{A}_{1}\right)$, $\left(\mathcal{A}_{2}\right)$ and $\left(\mathcal{A}_{3}\right)^{\prime}$ be satisfied and $p>2, q>\max \left\{p, \frac{p-1}{p-2}\right\}$. Then in the case

$$
G(s, u) \leq 0
$$

for each $\alpha \in\left(\lambda_{1}^{-1}, \lambda_{2}^{-1}\right]$ the Hammerstein equation (1) has at least two non-trivial solutions in $L_{p}$.

Proof. Since $\alpha \in\left(\lambda_{1}^{-1}, \lambda_{2}^{-1}\right)$ the form $\mathcal{E}_{\alpha}$ is negative definite on the one-dimensional subspace $E_{1}=\operatorname{Span}\left\{e_{1}\right\}$ and non-negative definite on its orthogonal complement in $E$. Set

$$
\mathcal{M}_{\alpha}^{+}=\left\{h \in E \mid \mathcal{E}_{\alpha}(h) \leq 0 \text { and }\left\langle e_{1}, h\right\rangle \geq 0\right\} .
$$

Clearly, $\mathcal{M}_{\alpha}^{+}$is convex and closed and hence weakly closed. By Theorem $1, \varphi$ attains its infimum on $\mathcal{M}_{\alpha}^{+}$at some point $x^{+}$. Since $\alpha>\lambda_{1}$ and $e_{1} \in E \cap L_{\frac{p}{q}+1}$ by Lemma 7 we have $\delta^{2} \varphi\left(0, e_{1}\right)=\mathcal{E}_{\alpha}\left(e_{1}\right)<0$ and, since $e_{1} \in \mathcal{M}_{\alpha}^{+}, \inf _{\mathcal{M}_{\alpha}^{+}} \varphi=\varphi\left(x^{+}\right)<0$. Hence $x^{+} \neq 0$.

We have to verify that $x^{+} \neq 0$ is a local minimum of $\varphi$ on the whole space $E$. If so, Theorem 2 implies that $x^{+}$is a non-trivial solution of equation (1). Clearly, it suffices to show that $x^{+}$lies in the interior of the set $\mathcal{M}_{\alpha}^{+}$. Suppose that the opposite is true, that is $x^{+} \in \partial \mathcal{M}_{\alpha}^{+}$. In this case $\mathcal{E}_{\alpha}\left(x^{+}\right)=0$ and by (6) we have

$$
\varphi\left(x^{+}\right)=\mathcal{E}_{\alpha}\left(x^{+}\right)-\int_{\Omega} G\left(s, x^{+}(s)\right) d s>0
$$

which is a contradiction. Therefore it follows that $x^{+} \in \mathcal{M}_{\alpha}^{+}$is a non-trivial solution of equation (1).

Put $\mathcal{M}_{\alpha}^{-}=-\mathcal{M}_{\alpha}^{+}$. Clearly, $\mathcal{M}_{\alpha}^{+} \cap \mathcal{M}_{\alpha}^{-}=\{0\}$ and by the same argument we obtain another non-trivial solution $x^{-} \in \mathcal{M}_{\alpha}^{-}$

Remark 10. The previous theorem is the only multiplicity result that we know for equation (1) under the natural growth conditions. In the case of a smooth functional $\varphi$, that is in the case of the classical growth conditions many multiplicity results of other types were obtained using critical point techniques (see, for example, $[15,16]$ ). In the last few years some variants of "Non-Smooth" Critical Point Theory have been presented by different authors (see, for example, $[2,18]$ and the references therein). A variant adopted to the study of the energy functional $\varphi$ for equation (1) under the natural growth conditions was given in [13]. Unfortunately, the attempts to verify the compactness condition of Palais-Smale type for $\varphi$ were unsuccessful and the multiple solution problem for the Hammerstein equation (1) under the natural growth conditions is still open.

Remark 11. The results given in the paper can be generalised to Hammerstein equations with kernels having a finite number of negative eigenvalues and also to systems of Hammerstein equations in ideal spaces of vector functions (see [14]). 


\section{References}

[1] Appell, J. and P. P. Zabreiko: Nonlinear Superposition Operators. Cambridge: Univ. Press 1990.

[2] Canino, A.-M. and M. Degiovanni: Nonsmooth critical point theory and quasilinear elliptic equations. Top. Meth. Diff. Equ. and Incl., Montreal 1994 (NATO ASI Ser. C). Dordrecht: Kluwer 1995, pp. 1 - 50.

[3] Brezis, H. and F. Browder: Some properties of higher order Sobolev spaces. J. Math. Pures Appl. 61 (1982), 245 - 259.

[4] Degiovanni, M. and S. Zani: Euler Equation Involving Nonlinearities Without Growth Conditions. Pot. Anal. 5 (1996), 505 - 512.

[5] De Figueiredo, D. and J. P. Gossez: Un problème elliptique semi-linéaire sans conditions de croissance. C.R. Acad. Sci. Paris (Sér. I) Math. 308 (1989), 277 - 280.

[6] Golomb, M.: Zur Theory der nichtlinearen Integralgleichungen. Integralgleichungssysteme und alLgemeine Functionalgleichungen. Math. Zeit. 39 (1934), $45-75$.

[7] Hammerstein, A.: Nichtlineare Integralgleichungen nebst Anwendungen. Acta Math. 54 (1930), $117-176$.

[8] Krasnosel'skii, A. M. and M. A. Krasnosel'skii: On some class of equations with potential operators. Note di Mat. 11 (1991), 237 - 245.

[9] Krasnosel'skii, M. A.: Topological Methods in the Theory of Nonlinear Integral Equations (in Russian). Moscow: Gostechizdat 1956. Engl. transl.: New York: Macmillan 1964.

[10] Krasnosel'skii, M. A., Zabreiko, P. P., Pustyl'nik, E. I. and P. E. Sobolevskii: Integral Operators in Spaces of Summable Functions (in Russian). Moscow: Nauka 1966. Engl. transl.: Leyden: Noordhoff 1976.

[11] Krein, S. G. and Ju. I. Petunin: Scales of Banach spases (in Russian). Uspehi Mat. Nauk $21(1966) 2,89-168$.

[12] Moroz, V. B.: An approach to Hammerstein integral equations (in Russian). Dokl. Acad. Nauk Belarusi 4 (1995), 31 - 35.

[13] Moroz, V. B.: Critical points of subspace-differentiable functionals and Hammerstein equations. Thesis. Minsk: Belorussian State University 1995, 95 pp.

[14] Moroz, V. B. and P. P. Zabreiko: New theorems on the solvability of Hammerstein integral equations with potential non-linearities (in Russian). Diff. Uravn. 31 (1995), 690 - 695.

[15] Moroz, V. B. and P. P. Zabreiko: $A$ variant of the mountain-pass theorem and its applications to Hammerstein integral equations. Z. Anal. Anw. 15 (1996), 985 - 997.

[16] Moroz, V. B., Vignoli, A. and P. P. Zabreiko: On the three critical point theorem. Top. Meth. Nonlin. Anal. 11 (1998), $103-113$.

[17] Rothe, E. H.: Completely continuous scalars and variational methods. Ann. Math. 49 (1948), 265 - 278.

[18] Struwe, M.: Variational Methods. Berlin: Springer-Verlag 1990.

[19] Vainberg, M. M.: Variational methods for the study of non-linear operators (in Russian). Moscow: Gostechizdat 1956. Engl.transl.: San-Francisco: Holden Day 1964.

[20] Zabreiko, P. P.: On the theory of Integral Operators (in Russian). Thesis. Voronej: State University 1968.

[21] Zabreiko, P. P. and A. 1. Povolotskii: On the theory of Hammerstein equations (in Russian). Ukrain. Mat. Zh. 22 (1970), $150-162$. 
[22] Zabreiko, A. I. Povolotskii: On second solutions of Hammerstein equations (in Russian). Vest. Jaros. Univ. 2 (1973), $24-30$.

Received 13.07.1988; in revised form 03.02.1999 